\title{
On perfect powers in products with terms from arithmetic progressions
}

\author{
by
}

\author{
N. Saradha (Bombay)
}

1. Introduction. The purpose of this paper is to obtain certain extensions of a remarkable theorem of Erdős and Selfridge [3, Theorem 1] that a product of two or more consecutive positive integers is never a power. If $n(n+1) \ldots(n+k-1)=y^{l}$ for positive integers $k, l, n, y$ with $k \geq 2$ and $l \geq 2$, then $\operatorname{ord}_{p}(n(n+1) \ldots(n+k-1))$ is congruent to $0(\bmod l)$ for every prime $p$. Erdős and Selfridge derived their result from the following statement.

Theorem A (Erdös and Selfridge [3, Theorem 2]). Let $k \geq 3, l \geq 2$ and $n \geq 1$ be integers such that $n+k-1 \geq p^{(k)}$ where $p^{(k)}$ is the least prime satisfying the inequality $p^{(k)} \geq k$. Then there is a prime $p \geq k$ for which $\operatorname{ord}_{p}(n(n+1) \ldots(n+k-1))$ is not congruent to $0(\bmod l)$.

In an earlier paper $([2])$, Erdős had shown that the equation

$$
n(n+1) \ldots(n+k-1)=k ! y^{l}
$$

has no solution under necessary conditions (see Section 2).

Theorem B (Erdős [2]). Let $k \geq 4, l \geq 2, n \geq k+1$ and $y \geq 1$ be integers. Then

$$
\left(\begin{array}{c}
n+k-1 \\
k
\end{array}\right)=y^{l}
$$

does not hold.

We observe that Theorem B is not a consequence of Theorem A whenever $k$ is a prime. The goal of the present paper is to extend Theorems A and B. This extension has the following form. Let $n>0, l \geq 2, k \geq k_{0}$ and $t \geq t_{0}=t_{0}(k)$ be integers where $k_{0}$ and $t_{0}$ are explicitly given numbers. Let $d_{1}, \ldots, d_{t}$ be distinct integers in the interval $[0, k-1]$. Let $d \in \Lambda$ where $\Lambda$ is an explicitly given finite set of positive integers depending only on $k$ and $l$.

1991 Mathematics Subject Classification: Primary 11D61. 
Suppose that $\left(n+d_{1} d\right) \ldots\left(n+d_{t} d\right)$ is divisible by a prime exceeding $k$. Then there exists a prime $p>k$ for which $\operatorname{ord}_{p}\left(\left(n+d_{1} d\right) \ldots\left(n+d_{t} d\right)\right) \not \equiv 0(\bmod l)$. The precise statements will be given in the next section. As an application of our result we derive the following generalisations of the theorem of Erdös and Selfridge [3, Theorem 1] mentioned in the beginning and of Theorem B. For an integer $\nu>1$, we define $P(\nu)$ to be the greatest prime factor of $\nu$ and write $P(1)=1$.

Corollary 1. The equation

$$
\begin{array}{cl}
n(n+d) \ldots(n+(k-1) d)=y^{l} \quad & \text { in integers } 1 \leq d \leq 6, k \geq 3, l \geq 2, \\
& n \geq 1, y \geq 1 \text { with } \operatorname{gcd}(n, d)=1
\end{array}
$$

has no solution.

Corollary 2. The equation

$$
\begin{aligned}
n(n+d) \ldots(n+(k-1) d)=b y^{l} \quad & \text { in integers } 1 \leq d \leq 6, k \geq 4, \\
& P(b) \leq k, l \geq 2, \\
& n \geq 1, y \geq 1 \text { with } \operatorname{gcd}(n, d)=1
\end{aligned}
$$

has no solution provided that the left hand side of the equation is divisible by a prime exceeding $k$ whenever $d=1$.

2. Results. For an integer $\nu>1$, we define $p(\nu)$ and $\omega(\nu)$ to be the smallest prime factor of $\nu$ and the number of distinct prime factors of $\nu$, respectively, and we write $p(1)=1$ and $\omega(1)=0$. Let $b, d, k \geq 2, l \geq 2$, $n, t \geq 2$ and $y$ denote positive integers such that $P(b) \leq k$ and $\operatorname{gcd}(n, d)=1$. Further, we write $d_{1}, \ldots, d_{t}$ for distinct integers in the interval $[0, k-1]$. We set

$$
\begin{gathered}
k_{0}= \begin{cases}4 & \text { if } d=1, \\
3 & \text { if } d>1,\end{cases} \\
\alpha(k)=\left[\frac{(.0156) k}{\log k}\right], \quad \beta(k)=\left[\frac{(.0017) k}{\log k}\right]
\end{gathered}
$$

and

$$
t_{0} \geq \begin{cases}k & \text { for } k \leq 8, l \geq 3 \text { and for } k \leq 24, l=2 \\ k-1 & \text { for } 9 \leq k \leq 11380, l \geq 3 \text { and } \\ & \text { for } 25 \leq k<870, l=2 \\ k-\alpha(k) & \text { for } k \geq 870, l=2 \\ k-\beta(k) & \text { for } k>11380, l \geq 3\end{cases}
$$

We assume that

$$
k \geq k_{0}, \quad t \geq t_{0} .
$$

We shall follow the above notation throughout the paper. We prove 
Theorem 1. (a) Let $k \geq k_{0}, t \geq t_{0}$ and

$$
\left\{\begin{array}{l}
d \in\{1,2,3,4,6\} \text { and } l \geq 2, \text { or } \\
d \leq 120, d \text { even and } l \geq 5, \text { or } \\
d \leq 36, d \text { odd, } 3 \mid d \text { and } l \geq 5 .
\end{array}\right.
$$

Assume that $\left(n+d_{1} d\right) \ldots\left(n+d_{t} d\right)$ is divisible by a prime exceeding $k$. Then there exists a prime $p>k$ for which

$$
\operatorname{ord}_{p}\left(\left(n+d_{1} d\right) \ldots\left(n+d_{t} d\right)\right) \not \equiv 0(\bmod l) .
$$

(b) Let $d=5, k \geq 4$ and

$$
t \geq \begin{cases}k & \text { for } l=3, \quad k \leq 25 \\ t_{0} & \text { otherwise. }\end{cases}
$$

Suppose that $\left(n+d_{1} d\right) \ldots\left(n+d_{t} d\right)$ is divisible by a prime exceeding $k$. Then there exists a prime $p>k$ satisfying (5).

Theorem 1 is equivalent to saying that under the assumptions of Theorem 1 , the equation

$$
\left(n+d_{1} d\right) \ldots\left(n+d_{t} d\right)=b y^{l}
$$

does not hold. Theorem 1 with $d=1$ answers a question of Shorey and Tijdeman $([8, \S 1])$. Furthermore, it answers some of the problems raised by Erdös and Selfridge at the end of their paper [3]. We observe that the hypothesis that $\left(n+d_{1} d\right) \ldots\left(n+d_{t} d\right)$ is divisible by a prime exceeding $k$ is necessary in Theorem 1. Shorey and Tijdeman [9] showed that this hypothesis is satisfied whenever $t=k, d>1$ and $(n, d, k) \neq(2,7,3)$. It is known that $n(n+1)=2 y^{2}$ has infinitely many solutions. Further, we have $n(n+1)(n+2)=6 y^{2}$ if $n=48, y=140$. The equation $n(n+d)=y^{l}$ can always be solved with $n=n_{1}^{l}, d=\left(n_{1}+1\right)^{l}-n_{1}^{l}$ for any positive integer $n_{1}$. Thus we see that the assumption $k \geq k_{0}$ with $k_{0}$ as in (1) is necessary in Theorem 1(a). Theorem 1(b) with $k=3$ remains unproved. We shall derive Theorems A and B from Theorem 1 in Section 7. In view of the examples given above, the assumption $k \geq 4$ of Theorem B is necessary. Now we consider Theorem B with $k \geq 2, l \geq 2, n \leq k$ and $y \geq 1$. It is clear from the examples given above that the equation in Theorem $\mathrm{B}$ has solutions if $n \leq 4$. Further, by the relation $\left(\begin{array}{c}n+k-1 \\ k\end{array}\right)=\left(\begin{array}{c}n+k-1 \\ n-1\end{array}\right)$, we derive from Theorem $\mathrm{B}$ that the equation in Theorem $\mathrm{B}$ does not hold if $n \geq 5$.

When $k$ is large, better bounds than (4) can be obtained for $d$ so that the assertion of Theorem 1 is valid. We have

Theorem 2. Let $k \geq 11380, t \geq t_{0}$ and

$$
d \leq \begin{cases}(.3) k^{1 / 3} & \text { if } l=2, \\ (1.75) k^{1 / 3} & \text { if } l=3, \\ 295 k^{l-3} & \text { if } l \geq 5 .\end{cases}
$$


Suppose $\left(n+d_{1} d\right) \ldots\left(n+d_{t} d\right)$ is divisible by a prime $>k$. Then there exists a prime $p>k$ satisfying $(5)$.

If $k$ exceeds a large effectively computable absolute constant (unspecified), we refer to Shorey and Tijdeman [10] and Shorey and Nesterenko [7] for better bounds for $d$ and $t$, respectively.

I thank Professor T. N. Shorey for many useful discussions and Professor R. S. Bhalerao for helping me with the computers in the calculation of error terms in Lemma 7. I also thank Professor R. Tijdeman for helping me with the preparation of Section 1.

3. Basic lemmas. In this section, we prove lemmas for the proofs of Theorems 1 and 2. We first observe that there is no loss of generality in assuming that $l$ is a prime number, which we suppose throughout the paper. Also we assume that

$$
P\left(\left(n+d_{1} d\right) \ldots\left(n+d_{t} d\right)\right)>k
$$

and

(8) $\operatorname{ord}_{p}\left(\left(n+d_{1} d\right) \ldots\left(n+d_{t} d\right)\right) \equiv 0(\bmod l) \quad$ for every prime $p>k$.

We shall use the above assumptions (7) and (8) without any further reference in this section. By (8), we write

(9) $\quad n+d_{i} d=a_{i} x_{i}^{l}, \quad P\left(a_{i}\right) \leq k, \quad a_{i}$ is $l$ th power free for $1 \leq i \leq t$

and

(10) $n+d_{i} d=A_{i} X_{i}^{l}, \quad P\left(A_{i}\right) \leq k, \quad \operatorname{gcd}\left(\prod_{p \leq k} p, X_{i}\right)=1 \quad$ for $1 \leq i \leq t$.

Let $S=\left\{a_{i} \mid 1 \leq i \leq t\right\}$ and $S^{\prime}=\left\{A_{i} \mid 1 \leq i \leq t\right\}$. Let $t^{\prime}$ be the number of distinct elements of $S$. We order the distinct elements of $S$ as $a_{1}^{\prime}<a_{2}^{\prime}<\ldots<a_{t^{\prime}}^{\prime}$. Using an argument of Erdös ([3, Lemma 2]), we find that there exist sets $S_{1} \subset S$ and $S_{1}^{\prime} \subset S^{\prime}$ with $\left|S_{1}\right|$ and $\left|S_{1}^{\prime}\right|$ greater than or equal to $t-\pi(k)$ such that

$$
\prod_{a_{i} \in S_{1}} a_{i} \leq(k-1) ! \text { and } \prod_{A_{i} \in S_{1}^{\prime}} A_{i} \leq(k-1) ! .
$$

From (7) and (9) we have $n+(k-1) d \geq(k+1)^{l}$, which implies that

$$
n>k^{l} \quad \text { if } d \leq l k^{l-2} \text {. }
$$

We begin with a lemma on Stirling's formula, upper bounds for $\pi(x)$ and $\vartheta(x)=\sum_{p \leq x} \log p$ and a lower bound for the $n$th prime $p_{n}$, the proofs of which can be found in [5, p. 447] and [6, pp. 69, 71]. 
Lemma 1. For any integer $M>1$, we have

(i) $\log M !<\log \sqrt{2 \pi}+\left(M+\frac{1}{2}\right) \log M-M+\frac{1}{12 M}$,

(ii) $\log M !>\log \sqrt{2 \pi}+\left(M+\frac{1}{2}\right) \log M-M$,

(iii) $\pi(M)<\frac{M}{\log M}\left(1+\frac{3}{2 \log M}\right)$,

(iv) $\vartheta(M)<(1.01624) M$,

(v) $p_{M}>M \log M$.

The next lemma deals with the distinctness property of $a_{i}$ 's and $A_{i}$ 's.

Lemma 2. (a) Let $l \geq 2$ and $d \leq l k^{l-2}$. Then the $a_{i}$ for $1 \leq i \leq t$ and $A_{i}$ for $1 \leq i \leq t$ are distinct.

(b) Let $l=2$.

(i) If $k \geq 11380$ and $2^{\omega(d)} d^{2} \leq(.00039) k(\log k)^{2}$, then the $a_{i}$ for $1 \leq$ $i \leq t$ are distinct.

(ii) If $d=3$, then the number of distinct $a_{i}$ 's is at least $t, t-1, t-2$ according as $k=3,4 \leq k \leq 22, k \geq 23$.

(iii) If $d=4$, then the $a_{i}$ for $1 \leq i \leq t$ are distinct.

(iv) If $d=5$, then the number of distinct $a_{i}$ 's is at least $t-2, t-3$ according as $4 \leq k \leq 38, k \geq 39$. Further, if $n>\frac{25}{4} k^{2}-15 k+9$, then the $a_{i}$ for $1 \leq i \leq t$ are distinct.

(v) If $d=6$, then the number of distinct $a_{i}$ 's is at least $t-1$.

Proof. (a) Let $a_{i}=a_{j}$ for $1 \leq i, j \leq t$ and $i \neq j$. We may assume without loss of generality that $n+d_{i} d>n+d_{j} d$. Then $x_{i}>x_{j}$ and

$$
d k>d\left(d_{i}-d_{j}\right)=\left(n+d_{i} d\right)-\left(n+d_{j} d\right)=a_{j}\left(x_{i}^{l}-x_{j}^{l}\right)>l a_{j} x_{j}^{l-1} .
$$

Thus we derive from (12) that

$$
d k>l\left(a_{j} x_{j}^{l}\right)^{(l-1) / l} \geq l n^{(l-1) / l}>l k^{l-1},
$$

which is a contradiction. The proof for the distinctness of the $A_{i}$ 's is similar.

(b) (i) Let $k \geq 11380$ and $2^{\omega(d)} d^{2} \leq(.00039) k(\log k)^{2}$. By Lemma 2(a), we may assume that $d \geq 3$. By an argument of Shorey and Tijdeman [10, p. 315], we show that

$$
n+(k-1) d>\frac{(.0001) k^{3}(\log k)^{2}}{2^{\omega(d)}} .
$$

From $n+(k-1) d \geq(k+1)^{2}$ it follows that

$$
n+d_{\mu} d \geq(k+1)^{2} / 35 \text { for } k / 35 \leq d_{\mu}<k .
$$

Let $T_{1}=\left\{\mu \mid k / 35 \leq d_{\mu}<k, X_{\mu}=1\right\}$ and $T_{2}=\left\{\mu \mid k / 35 \leq d_{\mu}<k\right.$, 
$\left.X_{\mu} \neq 1\right\}$. By an argument of Erdős [3, Lemma 2], we have

$$
\left|T_{1}\right| \leq \frac{k \log k}{\log \frac{(k+1)^{2}}{35}}+\pi(k),
$$

which, by (3), (2) and Lemma 1(iii), implies that $\left|T_{2}\right|>(.2278) k$. For $\mu \in T_{2}$, we have $X_{\mu}>k$ and $X_{\mu}$ 's are pairwise distinct. Further, we may assume that $X_{\mu}$ is prime for $\mu \in T_{2}$, otherwise, (13) follows. Then we can find a subset $T_{3}$ of $T_{2}$ such that

$$
\left|T_{3}\right| \geq \frac{1}{35}(.2278) k
$$

and by Lemma $1(\mathrm{v})$, we get for $\mu \in T_{3}$,

$$
X_{\mu} \geq \frac{34}{35}(.2278) k \log \left(\frac{34}{35}(.2278) k\right),
$$

i.e.,

$$
X_{\mu} \geq(.1854) k \log k
$$

We argue as in [10, pp. 315-316] to conclude that for every $A_{\mu}$ with $\mu \in T_{3}$, there exist at most $2^{\omega(d)+1} i$ 's belonging to $T_{3}$ with $A_{i}=A_{\mu}$. Thus there are at least $(.0032) k / 2^{\omega(d)}$ distinct $A_{i}$ 's. Hence

$$
n+(k-1) d \geq \frac{(.0032)(.1854)^{2} k^{3}(\log k)^{2}}{2^{\omega(d)}},
$$

which implies (13).

Now we proceed to show that the $a_{i}$ 's, for $1 \leq i \leq t$ are distinct. Let $a_{i}=a_{j}$ for $1 \leq i, j \leq t$ with $i \neq j$. We assume without loss of generality that $x_{i}>x_{j}$. By (13), we have

$$
\begin{aligned}
k d & >a_{i} x_{i}^{2}-a_{j} x_{j}^{2} \geq a_{j}\left(\left(x_{j}+1\right)^{2}-x_{j}^{2}\right)>2 a_{j} x_{j} \geq 2\left(a_{j} x_{j}^{2}\right)^{1 / 2} \\
& >2\left(\frac{(.0001) k^{3}(\log k)^{2}}{2^{\omega(d)}}-k d\right)^{1 / 2}
\end{aligned}
$$

which implies that

$$
2^{\omega(d)} d^{2}\left(1+\frac{4}{k d}\right)>(.0004) k(\log k)^{2} .
$$

Since $k \geq 11380$ and $d \geq 3$, it follows that $2^{\omega(d)} d^{2}>(.00039) k(\log k)^{2}$. This contradiction proves the distinctness of $a_{i}$.

For the proofs of (ii) to (v) we suppose $a_{i}=a_{j}$ for $1 \leq i, j \leq t$ and $i \neq j$. We assume without loss of generality that $n+d_{i} d>n+d_{j} d$ and hence $x_{i}>x_{j}$. Let $x_{i}=x_{j}+h$ for some positive integer $h$. Then

$$
\begin{aligned}
(k-1) d & \geq\left(d_{i}-d_{j}\right) d=\left(n+d_{i} d\right)-\left(n+d_{j} d\right) \\
& =a_{j}\left(x_{i}^{2}-x_{j}^{2}\right)=a_{j}\left(\left(x_{j}+h\right)^{2}-x_{j}^{2}\right)
\end{aligned}
$$




$$
\begin{aligned}
& =2 h a_{j} x_{j}+a_{j} h^{2}=2 h a_{j}^{1 / 2}\left(a_{j} x_{j}^{2}\right)^{1 / 2}+a_{j} h^{2} \\
& \geq 2 h a_{j}^{1 / 2} n^{1 / 2}+a_{j} h^{2} .
\end{aligned}
$$

(ii) Let $d=3$. From $n+(k-1) 3 \geq(k+1)^{2}$, it follows that $n \geq k^{2}-k+4$. We use this in (14) to get $h=1, a_{j} \leq 2$. Since $h=1$ the number of $i$ with $a_{i}=a_{j}$ and $i \neq j$ is at most one. If $a_{i}=a_{j}=2$, it follows from (14) that $k^{2}-22 k-7 \geq 0$, which implies that $k \geq 23$. Similarly, if $a_{i}=a_{j}=1$, we get $k \geq 4$. The result follows.

(iii) Let $d=4$. Since $a_{i}$ for $1 \leq i \leq t$ are odd, it follows from $\left(d_{i}-d_{j}\right) 4=$ $a_{j} h\left(2 x_{j}+h\right)$ that $h$ is even. We have $n \geq k^{2}-2 k+5$, which is used in (14) to give $h \leq 1$, a contradiction.

(iv) Let $d=5$. We have $n \geq k^{2}-3 k+6$. We use this in (14) to get $h \leq 2$. We observe from (14) that for $h=2, a_{j}=1$ and for $h=1, a_{j} \in\{1,2,3,4,6\}$, $a_{i}=a_{j}=6$ holds only for $k \geq 39$. Further, it follows from (14) that when $h=1$, we have $2 x_{j}+1 \equiv 0(\bmod 5)$. Thus $x_{j} \equiv 2(\bmod 5)$ implying that $n \equiv n+d_{j} 5=a_{j} x_{j}^{2} \equiv-a_{j}(\bmod 5)$. Thus $a_{j}$ belongs to $\{1,6\}$ or $\{2\}$ or $\{3\}$ or $\{4\}$. Now, the first part of the assertion follows easily. The second part is an easy consequence of (14).

(v) Let $d=6$. Here $a_{i}$ for $1 \leq i \leq t$ are odd and $h$ is even. Further, $n \geq k^{2}-4 k+7$ and it follows from (14) that $h=2, a_{j}=1$, which proves the result.

As an immediate consequence of (i) of Lemma 2(b), we get

Corollary 3 . Let $l=2, k \geq 11380$ and $d \leq(.3) k^{1 / 3}$. Then the $a_{i}$ for $1 \leq i \leq t$ are distinct.

In the next lemma, we improve (12) for $l \geq 3$ and $k \geq 9$.

LEMmA 3. Let $l \geq 3, k \geq 9$ and $d \leq l k^{l-2}$. Then

$$
n> \begin{cases}\gamma(k, l) k^{l} & \text { if } d \text { is odd, } \\ (2 \gamma(k, l)-1) k^{l} & \text { if } d \text { is even, }\end{cases}
$$

where $\gamma(k, l)=t-\pi(k)-k / l$.

Proof. By Lemma 2(a), we see that the $A_{i}$ for $1 \leq i \leq t$ are distinct. Further, from (11) and (12) we observe that

$$
\left|\left\{A_{i} \mid X_{i}=1,1 \leq i \leq t\right\}\right| \leq \frac{k \log k}{\log n}+\pi(k) \leq \frac{k}{l}+\pi(k) .
$$

Thus the set $\left\{A_{i} \mid X_{i} \neq 1,1 \leq i \leq t\right\}$ has cardinality $\geq \gamma(k, l)$. Also, for every $A_{i}$ in this set, $X_{i} \geq k+1$. We note that $A_{i}$ 's are odd if $d$ is even. Hence from the distinctness of $A_{i}$ 's it follows that

$$
n+(k-1) d \geq \begin{cases}\gamma(k, l)(k+1)^{l} & \text { if } d \text { is odd }, \\ (2 \gamma(k, l)-1)(k+1)^{l} & \text { if } d \text { is even. }\end{cases}
$$


Using (3) and Lemma 1(iii), we check that $\gamma(k, l) \geq 1$ for $k \geq 9$. The result now follows since $d \leq l k^{l-2}$.

Lemma 4. Let $l \geq 3$, and $k \geq 9$ whenever $l=3, d>1$. Suppose $l^{\prime}$ is a positive integer satisfying

$$
l^{\prime} \leq \begin{cases}l-1 & \text { if } d=1 \text { or } l=3 \\ l-2 & \text { if } d>1 \text { and } l \geq 5\end{cases}
$$

and

$$
d \leq \begin{cases}\frac{3}{2}(\gamma(k, 3))^{1 / 3}-\frac{1}{2 k} & \text { if } l=3, d \text { odd }, \\ \frac{3}{2}(2 \gamma(k, 3)-1)^{1 / 3}-\frac{1}{2 k} & \text { if } l=3, \text { d even }, \\ k^{l-l^{\prime}-1} & \text { if } l \geq 5 .\end{cases}
$$

Then the ratio of any two products $a_{i_{1}} \ldots a_{i_{l^{\prime}}}$ and $a_{j_{1}} \ldots a_{j_{l^{\prime}}}$ corresponding to distinct $l^{\prime}$-tuples $\left(i_{1}, \ldots, i_{l^{\prime}}\right)$ and $\left(j_{1}, \ldots, j_{l^{\prime}}\right)$ with $1 \leq i_{1} \leq \ldots \leq i_{l^{\prime}} \leq t$ and $1 \leq j_{1} \leq \ldots \leq j_{l^{\prime}} \leq t$ is not an lth power of a rational number.

Proof. The assumption on $d$ implies that $d \leq l k^{l-2}$. Thus (12) and Lemma 3 are valid. Let $1 \leq i_{1} \leq \ldots \leq i_{l^{\prime}} \leq t$ and $1 \leq j_{1} \leq \ldots \leq j_{l^{\prime}} \leq t$ with $\left(i_{1}, \ldots, i_{l^{\prime}}\right) \neq\left(j_{1}, \ldots, j_{l^{\prime}}\right)$ and

$$
a_{i_{1}} \ldots a_{i_{l^{\prime}}}=a_{j_{1}} \ldots a_{j_{l^{\prime}}}\left(t_{1} / t_{2}\right)^{l}
$$

where $t_{1}$ and $t_{2}$ are positive integers with $\operatorname{gcd}\left(t_{1}, t_{2}\right)=1$. We put

$$
A=\frac{a_{i_{1}} \ldots a_{i_{l^{\prime}}}}{t_{1}^{l}}=\frac{a_{j_{1}} \ldots a_{j_{l^{\prime}}}}{t_{2}^{l}}
$$

We note that $A$ is a positive integer. First, we show that

$$
\left(n+d_{i_{1}} d\right) \ldots\left(n+d_{i_{l^{\prime}}} d\right) \neq\left(n+d_{j_{1}} d\right) \ldots\left(n+d_{j_{l^{\prime}}} d\right) .
$$

Suppose (16) does not hold. Then we cancel any term on the left hand side which equals some term on the right hand side. There remains at least one term on the left hand side, say, $n+d_{i_{1}} d$. We note that for $1 \leq r \leq l^{\prime}$, $\operatorname{gcd}\left(n+d_{i_{1}} d, n+d_{j_{r}} d\right) \leq k$ since $\operatorname{gcd}(n, d)=1$. Thus

$$
n+d_{i_{1}} d \leq \operatorname{gcd}\left(n+d_{i_{1}} d, n+d_{j_{1}} d\right) \ldots \operatorname{gcd}\left(n+d_{i_{1}} d, n+d_{j_{l^{\prime}}} d\right) \leq k^{l^{\prime}}
$$

which, by (12), gives a contradiction. Thus (16) holds.

We may assume without loss of generality that

$$
\left(n+d_{i_{1}} d\right) \ldots\left(n+d_{i_{l^{\prime}}} d\right)>\left(n+d_{j_{1}} d\right) \ldots\left(n+d_{j_{l^{\prime}}} d\right),
$$

i.e.,

$$
a_{i_{1}} \ldots a_{i_{l^{\prime}}}\left(x_{i_{1}} \ldots x_{i_{l^{\prime}}}\right)^{l}>a_{j_{1}} \ldots a_{j_{l^{\prime}}}\left(x_{j_{1}} \ldots x_{j_{l^{\prime}}}\right)^{l} .
$$

Hence by (15), we get $A x^{l}>A y^{l}$ where $x=t_{1} x_{i_{1}} \ldots x_{i_{l^{\prime}}}$ and $y=t_{2} x_{j_{1}} \ldots$ $\ldots x_{j_{l^{\prime}}}$. So $x>y$. Thus

$$
\begin{aligned}
\left(n+d_{i_{1}} d\right) & \ldots\left(n+d_{i_{l^{\prime}}} d\right)-\left(n+d_{j_{1}} d\right) \ldots\left(n+d_{j_{l^{\prime}}} d\right) \\
& \geq A\left((y+1)^{l}-y^{l}\right)>l A y^{l-1}>l\left(A y^{l}\right)^{(l-1) / l}>l n^{(l-1) l^{\prime} / l} .
\end{aligned}
$$


On the other hand, using (12), $d \leq k^{l-l^{\prime}-1}$ if $l \geq 5$ and $d \leq \frac{3}{2} k^{1 / 3}$ if $l=3$, we get

$$
\begin{aligned}
\left(n+d_{i_{1}} d\right) \ldots & \left(n+d_{i_{l^{\prime}}} d\right)-\left(n+d_{j_{1}} d\right) \ldots\left(n+d_{j_{l^{\prime}}} d\right) \\
& <(n+k d)^{l^{\prime}}-n^{l^{\prime}}=l^{\prime} n^{l^{\prime}-1} k d+\left(\begin{array}{c}
l^{\prime} \\
2
\end{array}\right) n^{l^{\prime}-2}(k d)^{2}+\ldots \\
& \leq l n^{l^{\prime}-1} k d-n^{l^{\prime}-1} k d+\left(\begin{array}{c}
l^{\prime} \\
2
\end{array}\right) n^{l^{\prime}-2}(k d)^{2}\left\{1+\frac{l^{\prime} k d}{3 n}+\ldots\right\} \\
& <l n^{l^{\prime}-1} k d-n^{l^{\prime}-1} k d+l^{\prime}\left(l^{\prime}-1\right) n^{l^{\prime}-2}(k d)^{2}<l n^{l^{\prime}-1} k d,
\end{aligned}
$$

which, together with the lower bound given above, implies that $n^{\left(l-l^{\prime}\right) / l}<$ $k d$. When $l=3$ from the upper bound and the lower bound inequalities we in fact get $k^{2} d^{2}+2 n k d>3 n^{4 / 3}$ if $l^{\prime}=2$ and $k d>3 n^{2 / 3}$ if $l^{\prime}=1$. Now we use (12) if either $d=1$ or $l \geq 5$, and Lemma 3 if $d>1, l=3$, to get a contradiction.

From Lemma 4 it is clear that the $a_{i} a_{j}$ for $1 \leq i \leq j \leq t$ are all distinct if either $l \geq 3, d=1$ or

$$
d \leq \begin{cases}(1.4)(\gamma(k, 3))^{1 / 3} & \text { if } l=3 \text { and } k \geq 9 \\ k^{l-3} & \text { if } l \geq 5\end{cases}
$$

This restriction on $d$ is relaxed in the following lemma.

Lemma 5. Let $l \geq 3$ and $k \geq 9$ whenever $d>1$. Assume that

$$
d \leq \frac{7}{5} \cdot 4^{1 / l}(\gamma(k, l))^{1-2 / l} k^{l-3} .
$$

Then the $a_{i} a_{j}$ for $1 \leq i, j \leq t$ are distinct.

P r o o f. We observe that $d$, as given in the lemma, implies that $d \leq l k^{l-2}$. Hence by Lemma 2(a), the $a_{i}$ for $1 \leq i \leq t$ are distinct. Suppose $a_{i} a_{j}=a_{r} a_{s}$ for $(i, j) \neq(r, s)$ with $1 \leq i, j \leq t, 1 \leq r, s \leq t$ and $a_{i} \leq a_{j}, a_{r} \leq a_{s}$. Then we observe that $a_{i} a_{j}=a_{r} a_{s} \geq 4$. As shown in Lemma 4 , we have

$$
\left(n+d_{i} d\right)\left(n+d_{j} d\right) \neq\left(n+d_{r} d\right)\left(n+d_{s} d\right) .
$$

We may suppose that $\left(n+d_{i} d\right)\left(n+d_{j} d\right)>\left(n+d_{r} d\right)\left(n+d_{s} d\right)$. Thus $x_{i} x_{j}>x_{r} x_{s}$. Hence

$$
\begin{aligned}
2 k n d+k^{2} d^{2} & >\left(n+d_{i} d\right)\left(n+d_{j} d\right)-\left(n+d_{r} d\right)\left(n+d_{s} d\right) \\
& >l a_{r} a_{s}\left(x_{r} x_{s}\right)^{l-1}=l\left(a_{r} a_{s}\right)^{1 / l}\left(a_{r} x_{r}^{l} a_{s} x_{s}^{l}\right)^{(l-1) / l} \\
& >l\left(a_{r} a_{s}\right)^{1 / l} n^{2(l-1) / l}>l 4^{1 / l} n^{2(l-1) / l} .
\end{aligned}
$$


Thus we have

$$
\begin{aligned}
k^{2} d^{2} & >l 4^{1 / l} n^{2(l-1) / l}\left(1-\frac{2 k d}{l 4^{1 / l} n^{1-2 / l}}\right) \\
& >l 4^{1 / l} n^{2(l-1) / l}\left(1-\frac{2 k d}{3 \cdot 4^{1 / l} n^{1-2 / l}}\right) .
\end{aligned}
$$

For $d=1$, we use (12) to get a contradiction. Thus we may assume that $d>1$. Using Lemma 3 and our assumption on $d$ we get

$$
k^{2} d^{2}>\frac{l}{15} 4^{1 / l}(\gamma(k, l))^{2-2 / l} k^{2 l-2}
$$

in which we apply the bound for $d$ and $l \geq 3$ to obtain

$$
\frac{735}{25}>\frac{l}{4^{1 / l}}(\gamma(k, l))^{2 / l} k^{2}>1.8898 k^{2+2 / l}\left(\frac{t}{k}-\frac{\pi(k)}{k}-\frac{1}{3}\right)^{2 / 3} .
$$

We use $t \geq t_{0},(3)$, the exact value of $\pi(k)$ for $k \leq 20$ and the upper bound for $\pi(k)$ from Lemma 1(iii) for $k>20$ to check that

$$
\left(\frac{t}{k}-\frac{\pi(k)}{k}-\frac{1}{3}\right)^{2 / 3}>.2311 .
$$

Thus we have $k^{2+2 / l} \leq 68$. This is a contradiction since $k \geq 9$. This proves the lemma.

We need the following graph theoretic lemma from [3].

Lemma 6. Suppose $G$ is a bipartite graph of $s$ white vertices and $r$ black vertices which contains no rectangles. Then the number of edges is at most $s+\left(\begin{array}{l}r \\ 2\end{array}\right)$.

We use the above lemma as follows. Let $x \geq 1$ be an arbitrary real number. We construct two sets $U$ and $V$ of positive integers $\leq x$ such that all positive integers $\leq x$ can be written as $u v$ with $u \in U$ and $v \in V$. We take $(U, V)$ to be the bipartite graph $G$ with black vertices as elements of $U$ and white vertices as elements of $V$. Let $\left\{c_{1}, \ldots, c_{h}\right\}$ be a set of positive integers $\leq x$ with the property that the $c_{i} c_{j}$ for $1 \leq i, j \leq h$ are distinct. We say that there is an edge between an element $u \in U$ and $v \in V$ if $u v=c_{i}$ for $1 \leq i \leq h$. By the distinctness of $c_{i} c_{j}$ 's it follows that $G$ has no rectangle. Thus it follows from Lemma 6 that $h \leq|V|+\left(\begin{array}{c}|U| \\ 2\end{array}\right)$.

Now we explain the construction of the sets $U$ and $V$. Let $2=p_{1}<$ $p_{2}<\ldots$ be the sequence of all primes. More generally, let $p_{1}^{\prime}<p_{2}^{\prime}<\ldots$ be the sequence of all primes coprime to $d$. Since $\operatorname{gcd}(n, d)=1$, we observe that $a_{1}, \ldots, a_{t}$ given by (9) are composed of primes $p_{1}^{\prime}, p_{2}^{\prime}, \ldots$ For positive integers $m$ and $T$, we denote by $U=U(m, T)$ the set of integers $\leq T$ which are composed of $p_{1}, \ldots, p_{m}$. We observe that $1 \in U$. Further, we understand that an empty product equals 1 . We construct a set $V$ as follows. With 
every prime $p_{i}, 1 \leq i \leq m$, we associate an integer $r_{i}(T)$ such that $p_{i} r_{i}(T)$ is the smallest integer $>T$ with $P\left(p_{i} r_{i}(T)\right)=p_{i}$. We put

$$
r_{m+1}(T)=1 / p_{m+1}, \quad V_{i}=\left\{p_{i} w \mid w \leq x / r_{i}(T), p\left(p_{i} w\right)=p_{i}\right\}
$$

for $1 \leq i \leq m$,

$$
V_{m+1}=\left\{w \mid w \leq x, p(w)=1 \text { or } p(w) \geq p_{m+1}\right\} \quad \text { and } \quad V=\bigcup_{i=1}^{m+1} V_{i} .
$$

Then we see that for $1 \leq i \leq m+1$,

$$
\begin{aligned}
\left|V_{i}\right| & =\left|\left\{w \mid w \leq \frac{x}{p_{i} r_{i}(T)}, \operatorname{gcd}\left(w, p_{1} \ldots p_{i-1}\right)=1\right\}\right| \\
& =\frac{\varphi\left(p_{1} \ldots p_{i-1}\right)}{p_{1} \ldots p_{i-1}}\left[\frac{x}{p_{i} r_{i}(T)}\right]+E_{i}
\end{aligned}
$$

where $E_{i}$ 's are error terms and $\varphi$ is the Euler totient function. Since $V_{1}, \ldots$ $\ldots, V_{m+1}$ are pairwise disjoint, we have

$$
|V|=\sum_{i=1}^{m+1}\left(\frac{\varphi\left(p_{1} \ldots p_{i-1}\right)}{p_{1} \ldots p_{i-1}}\left[\frac{x}{p_{i} r_{i}(T)}\right]+E_{i}\right) .
$$

We observe that if $X=p_{1} \ldots p_{i-1} X^{\prime}+z$ where $X=\left[x /\left(p_{i} r_{i}(T)\right)\right]$ and $0 \leq z<p_{1} \ldots p_{i-1}$ then $\left|V_{i}\right|=\varphi\left(p_{1} \ldots p_{i-1}\right) X^{\prime}+\varrho(z)$ where $\varrho(z)$ is the number of integers $\leq z$ and coprime to $p_{1} \ldots p_{i-1}$. Hence

$$
\left|V_{i}\right|=\frac{\varphi\left(p_{1} \ldots p_{i-1}\right)}{p_{1} \ldots p_{i-1}} X+\varrho(z)-\frac{\varphi\left(p_{1} \ldots p_{i-1}\right)}{p_{1} \ldots p_{i-1}} z .
$$

Thus we see from (17) that for $1 \leq i \leq m+1$,

$$
E_{i} \leq \frac{1}{p_{1} \ldots p_{i-1}} \max \left\{p_{1} \ldots p_{i-1} \varrho(z)-\varphi\left(p_{1} \ldots p_{i-1}\right) z\right\}
$$

where the maximum is taken over all $0 \leq z<p_{1} \ldots p_{i-1}$ with $\operatorname{gcd}\left(z, p_{1} \ldots p_{i-1}\right)=1$. To find this maximum, we first enumerate all the integers $<p_{1} \ldots p_{i-1}$ which are coprime to $p_{1} \ldots p_{i-1}$. This is done by the method of sieving. Given an integer $z<p_{1} \ldots p_{i-1}$, we test if $z$ is divisible by $p_{j}$ for $1 \leq j \leq i-1$. If at any stage, the test is positive, then $z$ is deleted. If the test fails for all $j, 1 \leq j \leq i-1$, then $z$ is retained. Thus we obtain integers $z_{1}<z_{2}<\ldots<z_{\delta_{i}}$ where $\delta_{i}=\varphi\left(p_{1} \ldots p_{i-1}\right)$ which are coprime to $p_{1} \ldots p_{i-1}$. Then we compute $p_{1} \ldots p_{i-1} \mu-\varphi\left(p_{1} \ldots p_{i-1}\right) z_{\mu}$ for $1 \leq \mu \leq \delta_{i}$ and take the maximum which depends only on $i$. Bounds for $E_{1}, \ldots, E_{6}$ already appear in [3]. Bounds for $E_{7}, \ldots, E_{11}$ have been calculated using DEC AXP 3000 / 800 OSF / 1V3.0 at the Tata Institute of Fundamental Research. The times taken for the calculation of $E_{10}$ and $E_{11}$ are about 4 minutes and about 2 hours 8 minutes respectively, while other calculations, 
put together, took less than a minute. We record in the following lemma the bounds for $E_{i}$ 's which may be of independent interest.

LEMMA 7.

$$
\begin{gathered}
E_{1} \leq 0, \quad E_{2} \leq \frac{1}{2}, \quad E_{3} \leq \frac{2}{3}, \quad E_{4} \leq \frac{14}{15}, \quad E_{5} \leq \frac{53}{35}, \quad E_{6} \leq \frac{194}{77}, \\
E_{7} \leq \frac{3551}{1001}, \quad E_{8} \leq \frac{92552}{17017}, \\
E_{9} \leq \frac{2799708}{323323}, \quad E_{10} \leq \frac{9747144}{676039}, \quad E_{11} \leq \frac{58571113}{2800733} .
\end{gathered}
$$

In the next lemma, we construct several sets $U$ and $V$ as described above by choosing $m$ and $T$ suitably which enable us to obtain good lower bounds for $a_{h}^{\prime}$ which sharpen considerably the ones given in Erdős and Selfridge [3, (15), (16)].

Lemma 8. Let $l \geq 3$ and $k \geq 9$ whenever $d>1$. Assume that $d \leq$ $\frac{7}{5} \cdot 4^{1 / l}(\gamma(k, l))^{1-2 / l} k^{l-3}$. Then $a_{h}^{\prime} \geq \mu(h-\nu)$ where $(\mu, \nu)$ equals

(i) $(1,0)$ for $h \leq 16$,

(ii) $(1.7777,7)$ for $17 \leq h \leq 57$,

(iii) $(2.2153,17)$ for $58 \leq h \leq 177$,

(iv) $(2.5484,38)$ for $178 \leq h \leq 281$,

(v) $(2.9205,69)$ for $282 \leq h \leq 800$,

(vi) $(3.32,157)$ for $801 \leq h \leq 1335$,

(vii) $(3.565,238)$ for $1336 \leq h \leq 1790$,

(viii) $(4.1135,445)$ for $1791 \leq h \leq 2617$,

(ix) $(4.2444,512)$ for $2618 \leq h \leq 3786$,

(x) $(4.3878,619)$ for $3787 \leq h \leq 5711$,

(xi) $(4.4964,742)$ for $5712 \leq h \leq 7491$,

(xii) $(4.6189,921)$ for $7492 \leq h \leq 9183$,

(xiii) $(4.6425,963)$ for $h \geq 9184$.

Proof. By Lemma 2(a), elements of $S$ are distinct. Hence $t^{\prime}=t$ and $a_{h}^{\prime} \geq h$ is valid for $1 \leq h \leq t$. (See the first line in Table 1.) By Lemma 5 , we find that $a_{i}^{\prime} a_{j}^{\prime}$ for $1 \leq i, j \leq t$ are distinct. Let $x \geq 1$ be an arbitrary real number. As explained earlier, we can use Lemma 6 to get an upper bound for the number of $a_{h}^{\prime}$ which are $\leq x$.

We illustrate below the construction of the sets $U$ and $V$ which yields (iii). We take $U$ to be the set of all integers $\leq 8$ and composed of only 2 and 3 . Thus $m=2, T=8, U=U(2,8)$ and $|U|=6$. Next, $r_{1}(t)=8, r_{2}(t)=3$ and $r_{3}(t)=1 / 5$. Further, we have $V=V_{1} \cup V_{2} \cup V_{3}$ with $V_{1}=\{2 w \mid 2 w \leq x / 8\}$, $V_{2}=\{3 w \mid 3 w \leq x / 3, p(3 w)=3\}$ and $V_{3}=\{w \mid w \leq x, p(w)=1$ or 
$p(w) \geq 5\}$. From (18) and Lemma 7, we get

$$
|V| \leq\left\{\frac{1}{16}+\frac{1}{18}+\frac{1}{3}\right\} x+\frac{7}{6}<(.4514) x+2 .
$$

Now, we show that every integer $\leq x$ is representable as $u v$ with $u \in U$ and $v \in V$. Let $x^{\prime}=2^{a} 3^{b} x^{\prime \prime} \leq x$ with $\left(x^{\prime \prime}, 6\right)=1$. We give below the value of $u$ in all possible cases. The value of $v$ is given by $x^{\prime} / u$. We have for $a \geq 3$, $u=8 ; a=2, u=4 ; a=1, u=6$ if $b \geq 1 ; a=1, u=2$ if $b=0 ; a=0$, $u=3$ if $b \geq 1 ; a=0, u=1$ if $b=0$.

Now, we use Lemma 6 to derive that the number of $a_{h}^{\prime}$ which are less than or equal to $x$ is bounded by (.4514) $x+17$. Taking $x=a_{h}^{\prime}$, we get $a_{h}^{\prime} \geq 2.2153(h-17)$. The proof of other values of $(\mu, \nu)$ are similar. We give below in Table 1 the values of $m$ and $T$ which are used to obtain the values of $(\mu, \nu)$ listed in (i) to (xiii) of the lemma. Also, we give the cardinalities of the respective sets $U$ and $V$.

Table 1

\begin{tabular}{cccccccc}
\hline Assertion No. & $m$ & $T$ & $r=|U|$ & $s=|V|$ & $\mu$ & $\nu$ & Least value of $h$ \\
\hline (i) & - & - & - & - & 1 & 0 & 1 \\
(ii) & 1 & 8 & 4 & $.5625 x+1$ & 1.7777 & 7 & 17 \\
(iii) & 2 & 8 & 6 & $.4514 x+2$ & 2.2153 & 17 & 58 \\
(iv) & 2 & 16 & 9 & $.3924 x+2$ & 2.5484 & 38 & 178 \\
(v) & 3 & 16 & 12 & $.3424 x+3$ & 2.9205 & 69 & 282 \\
(vi) & 4 & 24 & 18 & $.3012 x+4$ & 3.32 & 157 & 801 \\
(vii) & 5 & 27 & 22 & $.2805 x+7$ & 3.565 & 238 & 1336 \\
(viii) & 6 & 36 & 30 & $.2431 x+10$ & 4.1135 & 445 & 1791 \\
(ix) & 7 & 36 & 32 & $.2356 x+16$ & 4.2444 & 512 & 2618 \\
(x) & 8 & 39 & 35 & $.2279 x+24$ & 4.3878 & 619 & 3787 \\
(xi) & 9 & 42 & 38 & $.2224 x+39$ & 4.4964 & 742 & 5712 \\
(xii) & 10 & 46 & 42 & $.2165 x+60$ & 4.6189 & 921 & 7492 \\
(xiii) & 10 & 48 & 43 & $.2154 x+60$ & 4.6425 & 963 & 9184 \\
\hline
\end{tabular}

Let $m \geq 1$ be an integer. For $d=1$, we define $A_{m}=\left\{a_{h}^{\prime} \mid P\left(a_{h}^{\prime}\right) \leq p_{m}\right\}$ and $f(k, m)=\left|A_{m}\right|$. Since $t^{\prime}=t$, we have

$$
f(k, m) \geq t-\sum_{h \geq m+1}\left(\left[\frac{k}{p_{h}}\right]+\varepsilon_{h}\right):=f_{0}(k, m)
$$

where $\varepsilon_{h}=0$ if $p_{h}>k$ and for $p_{h} \leq k, \varepsilon_{h}=0$ or 1 according as $p_{h} \mid k$ or not for $h \geq m+1$. Further, we define $B_{m}=\left\{a_{h}^{\prime} \mid P\left(a_{h}^{\prime}\right) \leq p_{m}^{\prime}\right\}$ and $g(k, m)=\left|B_{m}\right|$. Then

$$
g(k, m) \geq t^{\prime}-\sum_{h \geq m+1}\left(\left[\frac{k}{p_{h}^{\prime}}\right]+\varepsilon_{h}^{\prime}\right):=g_{0}(k, m)
$$


where $\varepsilon_{h}^{\prime}=0$ if $p_{h}^{\prime}>k$ and for $p_{h}^{\prime} \leq k, \varepsilon_{h}^{\prime}=0$ or 1 according as $p_{h}^{\prime} \mid k$ or not for $h \geq m+1$. It is easily seen that $g_{0}(k, m) \geq f_{0}(k, m)$ whenever $t^{\prime}=t$. Suppose $d$ is divisible by either 2 or 3 . Then $p_{i}^{\prime} \geq p_{i+1}$ for $i \geq 2$. Thus for $m \geq 2$ and $t^{\prime}=t$ we get

$$
g_{0}(k, m-1) \geq f_{0}(k, m) \quad \text { if } 2 \mid d \text { or } 3 \mid d .
$$

As $k$ increases, $f_{0}(k, m)$ and $g_{0}(k, m)$ become $\leq 0$ and hence useless. For these values of $k$, we proceed as follows. Let $p_{1}<\ldots<p_{m_{1}} \leq k^{3 / 10}<$ $p_{m_{1}+1}<\ldots<p_{m_{1}+m_{2}} \leq \sqrt{k}$. For $d=1$, we define $A=\left\{a_{i}^{\prime} \mid P\left(a_{i}^{\prime}\right) \leq \sqrt{k}\right.$ and $a_{i}^{\prime}$ is divisible by at most one of the primes $p_{m_{1}+j}$ for $1 \leq j \leq m_{2}$ which divides $a_{i}^{\prime}$ only to the first power $\}$ and $F(k)=|A|$. Then we note that (see [3, p. 298])

$$
\begin{aligned}
F(k) \geq & t-\sum_{\sqrt{k}<p \leq k}\left(\left[\frac{k}{p}\right]+1\right)-\left\{\frac{k}{2}\left(\sum_{i=1}^{m_{2}} \frac{1}{p_{m_{1}+i}^{2}}+\left(\sum_{i=1}^{m_{2}} \frac{1}{p_{m_{1}+i}}\right)^{2}\right)\right\} \\
& -\left(\begin{array}{c}
m_{2}+1 \\
2
\end{array}\right) \\
:= & F_{0}\left(k, m_{1}, m_{2}\right) .
\end{aligned}
$$

For $d>1$, we let $p_{1}^{\prime}<\ldots<p_{m_{1}^{\prime}}^{\prime} \leq k^{3 / 10}<p_{m_{1}^{\prime}+1}^{\prime}<\ldots<p_{m_{1}^{\prime}+m_{2}^{\prime}}^{\prime} \leq \sqrt{k}$ be all the primes $\leq \sqrt{k}$ and coprime to $d$. We observe that $m_{1}^{\prime} \leq m_{1}$ and $m_{2}^{\prime} \leq m_{2}$. Further, for $m_{1} \geq 2, m_{1}^{\prime} \leq m_{1}-1$ if $2 \mid d$ or $3 \mid d$. We define $B=\left\{a_{i}^{\prime} \mid P\left(a_{i}^{\prime}\right) \leq \sqrt{k}\right.$ and $a_{i}^{\prime}$ is divisible by at most one of the primes $p_{m_{1}^{\prime}+j}^{\prime}$ for $1 \leq j \leq m_{2}^{\prime}$ which divides $a_{i}^{\prime}$ only to the first power $\}$ and $G(k)=|B|$. Then as before, we have

$$
G(k) \geq G_{0}\left(k, m_{1}^{\prime}, m_{2}^{\prime}\right)
$$

where $G_{0}\left(k, m_{1}^{\prime}, m_{2}^{\prime}\right)$ is got from the expression for $F_{0}\left(k, m_{1}, m_{2}\right)$ by replacing $t, m_{1}, m_{2}, p_{m_{1}+i}$ by $t^{\prime}, m_{1}^{\prime}, m_{2}^{\prime}, p_{m_{1}^{\prime}+i}^{\prime}$, respectively. When $t^{\prime}=t$, we have

$$
G_{0}\left(k, m_{1}^{\prime}, m_{2}^{\prime}\right) \geq F_{0}\left(k, m_{1}, m_{2}\right) .
$$

Following the argument of [3], we have

Lemma 9. Suppose the hypothesis of Lemma 4 holds. Then

(i) For $d=1, l \geq 3, m \geq 1, f(k, m) \geq 1$ and $F(k) \geq 1$, we have

$$
\left(\begin{array}{c}
f(k, m)+l-2 \\
l-1
\end{array}\right) \leq l^{m}
$$

and

$$
\left(\begin{array}{c}
F(k)+l-2 \\
l-1
\end{array}\right) \leq l^{m_{1}}\left(\begin{array}{c}
l+m_{2}-1 \\
l-1
\end{array}\right) .
$$


(ii) For $d>1, l \geq 3, m^{\prime} \geq 1, g\left(k, m^{\prime}\right) \geq 1$ and $G(k) \geq 1$, we have

$$
\left(\begin{array}{c}
g\left(k, m^{\prime}\right)+l^{\prime}-1 \\
l^{\prime}
\end{array}\right) \leq l^{m^{\prime}}
$$

and

$$
\left(\begin{array}{c}
G(k)+l^{\prime}-1 \\
l^{\prime}
\end{array}\right) \leq l^{m_{1}^{\prime}}\left(\begin{array}{c}
l^{\prime}+m_{2}^{\prime} \\
l^{\prime}
\end{array}\right) .
$$

The next result was quoted by Erdös in [2]. This result was proved by A. Meyl in 1878. We refer to [1, p. 25] for further details. This result is independent of the assumptions (7) and (8).

LEMMA 10. The only solutions of the equation

$$
n(n+1)(n+2)=6 y^{2}
$$

in integers $n>1, y>1$ are $n=2, y=2 ; n=48, y=140$.

4. An algorithm. In this section we provide an algorithm to test that (7) does not hold whenever (8) holds.

Algorithm. Let $c, d, k, l$ be given with $c<k^{l}$ and $d<(k+1)^{l} /(k-1)$.

S te p 1. Find all primes $q_{1}, \ldots, q_{\theta}, q_{\theta+1}, \ldots, q_{\theta+\eta}$ which are coprime to $d$ and such that $q_{1}<\ldots<q_{\theta} \leq k<q_{\theta+1}<\ldots<q_{\theta+\eta}$ and $q_{\theta+i}^{l}<c k^{l}$ for $1 \leq i \leq \eta$.

Step 2. For $1 \leq h \leq \eta$, form the sets $D_{h}=\left\{q_{1}^{\beta_{1}} \ldots q_{\theta}^{\beta_{\theta}} q_{\theta+h}^{l} \mid q_{1}^{\beta_{1}} \ldots q_{\theta}^{\beta_{\theta}} q_{\theta+h}^{l} \leq c k^{l}\right.$ for integers $\left.\beta_{i} \geq 0,1 \leq i \leq \theta\right\}$ and let $D=\bigcup_{h=1}^{\eta} D_{h}$.

Step 3. For every $q \in D$, we find some $j=j(q)$ with $1 \leq j \leq k-1$ such that $P(q+j d)$ and $P(q-(k-j) d)$ are $>q_{\theta+\eta}$.

In Step 3 we observe that $q-(k-j) d$ is positive since $q \geq(k+1)^{l}$ and $d<(k+1)^{l} /(k-1)$. The above Algorithm yields the following result.

LEMMA 11. Let $c, d, k, l, n$ and $t$ be given such that $t=k, n+(k-1) d \leq$ $c k^{l}, c<k^{l}$ and $d<(k+1)^{l} /(k-1)$. If (8) and Step 3 hold, then (7) does not hold.

Proof. For any $p>k$, we observe from (8) that

$$
\operatorname{ord}_{p}(n(n+d) \ldots(n+(k-1) d))=0 \text { or } l
$$

since $c<k^{l}$. Further, we note that if $q_{\theta+h}$ with $1 \leq h \leq \eta$ divides a term in the product $n(n+d) \ldots(n+(k-1) d)$, then no other $q_{\theta+h^{\prime}}$ for $h^{\prime} \neq h, 1 \leq h^{\prime} \leq \eta$ divides the same term. Thus every term $n+i d$ is of the form $q^{\prime} q_{\theta+h}^{l}$ or $q^{\prime}$ where $P\left(q^{\prime}\right) \leq q_{\theta}$. Thus

$$
P(n(n+d) \ldots(n+(k-1) d)) \leq q_{\theta+\eta} .
$$


Suppose $n+i d=q$ for some $i$ with $0 \leq i \leq k-1$ and $q \in D$. Then $n+(i+j) d=q+j d$ is a term in the product $n(n+d) \ldots(n+(k-1) d)$ if $i+j \leq k-1$. Therefore $P(n(n+d) \ldots(n+(k-1) d))>q_{\theta+\eta}$ if $i+j \leq k-1$. This is a contradiction. Let $i+j>k-1$. Then $n+(i+j-k) d=q-(k-j) d$. Since $0 \leq i+j-k \leq k-2$, we see that $n+(i+j-k) d$ is a term in the product $n(n+d) \ldots(n+(k-1) d)$. Therefore $P(n(n+d) \ldots(n+(k-1) d))>q_{\theta+\eta}$, which is a contradiction. Hence $n+i d \notin D$ for $0 \leq i \leq k-1$. This implies that $P(n(n+d) \ldots(n+(k-1) d)) \leq q_{\theta} \leq k$, which contradicts $(7)$.

5. Proof of Theorems 1 and 2 for $l=2$. We assume that (7) and (8) hold and we arrive at a contradiction if either the assumptions of Theorem 1 or of Theorem 2 hold. Thus Lemma 2 and Corollary 3 are valid and we conclude that the $a_{i}^{\prime}$ for $1 \leq i \leq t$ are distinct whenever $d \in\{1,2,4\}$ or $d \leq(.3) k^{1 / 3}$ with $k \geq 11380$. Further, they are square free. We observe that out of 36 consecutive integers there are at most 24 square free integers. Writing the $h$ th square free integer, say $s_{h}$, as $s_{h}=36 f_{1}+f_{2}$ with $0 \leq f_{2}<36$, we find that

$$
h \leq 24 f_{1}+\min \left(f_{2}, 24\right) \leq \frac{2}{3}\left(s_{h}-f_{2}\right)+\min \left(f_{2}, 24\right) .
$$

Thus $s_{h} \geq \frac{3}{2}(h-8)$. Hence for $t \geq 9$,

$$
\begin{aligned}
\prod_{i=1}^{t} a_{i}^{\prime} & \geq(1 \cdot 2 \cdot 3 \cdot 5 \cdot 6 \cdot 7 \cdot 10 \cdot 11)\left(\frac{3}{2}\right)^{t-8}(t-8) ! \\
& =138600\left(\frac{3}{2}\right)^{t-8}(t-8) ! .
\end{aligned}
$$

By following the argument of $[10$, p. 323], we also have

$$
\prod_{i=1}^{t} a_{i}^{\prime} \leq 2^{\frac{8}{3}-\frac{2 k}{3}+\frac{2 \log k}{\log 2}} 3^{\frac{9}{4}-\frac{k}{4}+\frac{2 \log k}{\log 3}(k-1) !} \prod_{p \leq k} p .
$$

From Lemma 1(iv), we have $\prod_{p \leq k} p \leq(2.78)^{k}$, which implies that

$$
\prod_{i=1}^{t} a_{i}^{\prime} \leq(75.23) k^{4}(k-1) !(1.34)^{k} .
$$

Let $k \geq 870$. Since $t \geq k-\alpha(k)$, we deduce from (28) and (29) that

$$
(71.88)(1.119)^{k} \leq k^{\alpha(k)+11}(1.5)^{\alpha(k)} .
$$

Since $\alpha(k) \leq(.0156) k / \log k$, by taking the $k$ th root on both sides of $(30)$, we find that (30) is not satisfied. Let $680 \leq k \leq 869$. Then $t \geq k-1$ and we see from (28) and (29) that $(47.92)(1.119)^{k} \leq k^{12}$, which is not possible. Thus we may assume that $k<680$. 
First, we consider the case $d=1$ and $k<680$. Since the $a_{i}^{\prime}$ are distinct and square free, we have $f(k, m) \leq 2^{m}$ for all $m$. Thus if $f_{0}(k, m) \geq 2^{m}+1$ for some $m$, we get a contradiction by (19). We check using (19) with $t=k$ for $k \leq 24, t=k-1$ for $25 \leq k<680$ that

$$
\left\{\begin{array}{l}
f_{0}(k, 2) \geq 5 \text { for } 9 \leq k \leq 22, f_{0}(k, 3) \geq 9 \text { for } 23 \leq k \leq 78 \\
f_{0}(k, 4) \geq 17 \text { for } 79 \leq k \leq 276, f_{0}(k, 5) \geq 33 \text { for } 277 \leq k \leq 493 \\
f_{0}(k, 6) \geq 65 \text { for } 494 \leq k<680
\end{array}\right.
$$

Here and at many other places checkings were done using PARI-GP. We are left with $4 \leq k \leq 8$. Then $t=k$. We use repeatedly the following two facts without mention to deal with these values of $k$. The product of four consecutive integers is never a square (see [3, p. 300]). There are at most four terms from $\left\{n+d_{i} \mid 1 \leq i \leq t\right\}$ with $a_{i}^{\prime}$ composed of only 2 and 3 , and they must belong to $\left\{y_{1}^{2}, 2 y_{2}^{2}, 3 y_{3}^{2}, 6 y_{4}^{2}\right\}$ for some positive integers $y_{1}, y_{2}, y_{3}$ and $y_{4}$, since $a_{i}^{\prime}$ are distinct and square free. Thus the product of the four terms is a square. We observe that $k \neq 4$. Let $k=5$. Then $P\left(a_{i}^{\prime}\right) \leq 5$. Here we may assume that $5 \nmid n$ and $5 \nmid(n+4)$. Suppose $5 \mid(n+2)$. Then $n(n+1)(n+$ $3)(n+4)=X_{1}^{2}$ for some positive integer $X_{1}$. Thus $\left(n^{2}+4 n+\frac{3}{2}\right)^{2}-\frac{9}{4}=X_{1}^{2}$. This is impossible. Let $5 \mid(n+1)$. Then $n \equiv 4(\bmod 5)$. Hence $n=y_{1}^{2}$ or $6 y_{4}^{2}$. Let $n=y_{1}^{2}$. Then $n+2=6 y_{4}^{2}, n+3=2 y_{2}^{2}$ or $3 y_{3}^{2}$, which is impossible since $n+2$ and $n+3$ are coprime. Let $n=6 y_{4}^{2}$. Then $n+2=y_{1}^{2}, n+3=3 y_{3}^{2}$ and $n+4=2 y_{2}^{2}$. This means $(n+2)(n+3)(n+4)=6 X_{2}^{2}$ for some positive integer $X_{2}$, which is not possible by Lemma 10. Let $5 \mid(n+3)$. Arguing as before, we have $n=2 y_{2}^{2}, n+1=3 y_{3}^{2}, n+2=y_{1}^{2}, n+4=6 y_{4}^{2}$ implying $n(n+1)(n+2)=6 X_{3}^{2}$ for some positive integer $X_{3}$, which by Lemma 10 implies that $n=2$. In this case $P(n(n+1)(n+2)(n+3)(n+4))=5$, contradicting our assumption (7).

Thus $k \neq 5$. For $k=6$, we observe that 5 divides $n$ and $n+5$. But this means $(n+1)(n+2)(n+3)(n+4)$ is a square, which is impossible. Let $k=7$. Then we observe that there exist distinct $i_{1}, i_{2}$, and $i_{3}$ between 0 and 6 such that $7\left|\left(n+i_{1}\right), 5\right|\left(n+i_{2}\right)$ and $5 \mid\left(n+i_{3}\right)$. We consider the possibility $7|(n+1), 5| n, 5 \mid(n+5)$. Then $n \equiv 6(\bmod 7)$. Therefore $\{n+4, n+6\}=\left\{3 y_{3}^{2}, 6 y_{4}^{2}\right\}$, which is impossible. The other possibilities can be excluded similarly. Let $k=8$. Then we derive that $7|n, 7|(n+7)$ and $5|(n+1), 5|(n+6)$. Consequently, $(n+2)(n+3)(n+4)(n+5)$ is a square, which is not possible.

Let $d \in\{2,4\}$ and $k<680$. Then the $a_{i}^{\prime}$ are odd and square free integers. Consequently, we derive that $k \geq 9$. We observe from (21) and (20) that for $m \geq 2, f_{0}(k, m) \leq g_{0}(k, m-1) \leq g(k, m-1) \leq 2^{m-1}$, which is not possible by (31).

Let $d \in\{3,6\}$. By (ii) and (v) of Lemma 2(b), there are at least $t-2$ distinct $a_{i}^{\prime}$. Further, they are square free integers. We proceed as at the 
beginning of this section with $t$ replaced by $t-2$ to obtain $k<900$. Since $t^{\prime} \geq t-2$ we deduce from (20) and (19) for $m \geq 2$ that $f_{0}(k, m)-2 \leq$ $g_{0}(k, m-1) \leq g(k, m-1) \leq 2^{m-1}$, which, together with (31), implies that $3 \leq k \leq 8$ and $680 \leq k<900$. We consider $680 \leq k<900$. We check that $f_{0}(k, 6) \geq 35$, which is sufficient to get a contradiction. Let $4 \leq k \leq 8$. By (ii) and (v) of Lemma 2(b), there are at least $t-1$ distinct $a_{i}^{\prime}$. Hence the number of $a_{i}^{\prime}$ composed only of $p_{1}^{\prime}$ is $\geq 3$ while at most two such $a_{i}^{\prime}$ are possible. If $k=3, d=3$, all the three $a_{i}^{\prime}$ are distinct and composed of only the prime 2 , which is not possible. If $k=3, d=6$, by ( $\mathrm{v}$ ) of Lemma $2(\mathrm{~b})$, at least two $a_{i}^{\prime}$ are distinct. This is not possible since $P\left(a_{i}^{\prime}\right) \leq 3$ and $\operatorname{gcd}\left(a_{i}^{\prime}, 6\right)=1$.

Let $d=5$. By (iv) of Lemma 2(b), there are at least $t-3$ distinct, square free $a_{i}^{\prime}$. The argument at the beginning of this section with $t$ replaced by $t-3$ yields $k<1000$. From (20) and (19) we observe that for $m \geq 3$, $f_{0}(k, m)-3 \leq g_{0}(k, m-1) \leq g(k, m-1) \leq 2^{m-1}$ and hence by (31), we have $4 \leq k \leq 22$ and $680 \leq k<1000$. We check that $f_{0}(k, 6) \geq 36$ for $680 \leq k<1000$, which is sufficient to get a contradiction. Let $4 \leq k \leq 22$. The number of distinct $a_{i}^{\prime}$ is at least $t-2$. We observe that the number of $a_{i}^{\prime}$ composed of $p_{1}^{\prime}$ and $p_{2}^{\prime}$ is at least 5 for $9 \leq k \leq 22$ while this number cannot exceed 4 . Thus we may assume that $4 \leq k \leq 8$. Suppose $n>\frac{25}{4} k^{2}-15 k+9$. Then by (iv) of Lemma 2(b), all $a_{i}^{\prime}$ are distinct and hence the number of $a_{i}^{\prime}$ composed of $p_{1}^{\prime}$ and $p_{2}^{\prime}$ is at least 5 for $5 \leq k \leq 8$, which is a contradiction. For $k=4$, we note that for $0 \leq i \leq 3, n+i 5 \in\left\{y_{1}^{2}, 2 y_{2}^{2}, 3 y_{3}^{2}, 6 y_{4}^{2}\right\}$ where $y_{1}, y_{2}, y_{3}, y_{4}$ are some positive integers. Hence $n(n+5)(n+10)(n+15)$ is a perfect square, say $X^{2}$. We put $Y=n^{2}+15 n+25$ to observe that $Y^{2}-X^{2}=625$. Since $\operatorname{gcd}(X, Y)=1$, we have $Y-X=1, Y+X=625$, which implies that $Y=313$, but $n^{2}+15 n+25=313$ has no solution in integers. Thus we may assume that $4 \leq k \leq 8$ and $n \leq \frac{25}{4} k^{2}-15 k+9$. Let $k=8$. Then $n+(k-1) d \leq 324$. We apply the Algorithm of Section 3 to get $c=5.07<8^{2}, \theta=3, \eta=3, q_{1}=2, q_{2}=3, q_{3}=7, q_{4}=11, q_{5}=13, q_{6}=17$ and $D=\left\{11^{2}, 2 \cdot 11^{2}, 13^{2}, 17^{2}\right\}$. We take $j=4$ for $q \in\left\{11^{2}, 17^{2}\right\}$ and $j=1$ for $q \in\left\{2 \cdot 11^{2}, 13^{2}\right\}$ to check Step 3. Hence by Lemma 11, assumption (7) does not hold, which is a contradiction. Thus $k \neq 8$. Here and in the sequel, checkings involving the Algorithm were done using Mathematica. We apply the above argument for $4 \leq k \leq 7$ to complete the proof for $d=5$. This concludes the proof for $l=2$.

6. Proof of Theorems 1 and $\mathbf{2}$ for $l \geq 3$. We assume that (7) and (8) hold and we arrive at a contradiction if either the assumptions of Theorem 1 or of Theorem 2 hold.

First we consider the case where $k \geq 11380$. Then

$$
d \leq \frac{7}{5} \cdot 4^{1 / l}(\gamma(k, l))^{1-2 / l} k^{l-3} .
$$


Hence Lemma 8 is valid. We set

$$
Q(k)=\prod_{h=1}^{\delta(k)} a_{h}^{\prime} \quad \text { where } \delta(k)=k-\beta(k)-\pi(k) .
$$

We use Lemmas 8, 1(i) and 1(ii) to get

$$
\begin{aligned}
\log Q(k) & \geq \log \left\{16 !(1.7777)^{41} \frac{50 !}{9 !} \ldots(4.6425)^{\delta(k)-9183} \frac{(\delta(k)-963) !}{8220 !}\right\} \\
& \geq 6227.23+(\log 4.6425) \delta(k)+\log (\delta(k)-963) ! \\
& \geq 6227.23+(1.5352) \delta(k)+\log (\delta(k)-963) ! .
\end{aligned}
$$

Thus

$$
Q(k)>k !
$$

is valid if

$$
6227.23+(1.5352) \delta(k)+\log (\delta(k)-963) !>\log k !,
$$

which, again by Lemma 1(i) and (ii), is valid if

$6227.23+(1.5352) \delta(k)$

$>\left(k+\frac{1}{2}\right) \log k-k-(\delta(k)-962.5) \log (\delta(k)-963)+\delta(k)-963+\frac{1}{12 k}$,

i.e.,

$$
\begin{aligned}
& 6227+(1.5352) \delta(k) \\
& >(\beta(k)+\pi(k)+963)(\log k-1)+(\delta(k)-962.5) \log \frac{k}{\delta(k)-963} .
\end{aligned}
$$

Let $k \geq 14250$. Then

$$
\log \frac{k}{\delta(k)-963}<.2092 \text { and } \pi(k)<\frac{1.157 k}{\log k}
$$

by Lemma 1(iii). Using these estimates we check that (33) and hence (32) are valid for $k \geq 14250$. Next, we use the exact value of $\pi(k)$ from [4] to see that (33) and therefore (32) is valid for $k=11380$. Thus we need to check (32) for $k \in[11381,14249]=: I$. We note that for $k \in I, \beta(k)=2$ and

$$
Q(k+1)= \begin{cases}Q(k) & \text { if } k+1 \text { is a prime, } \\ Q(k) a_{k-\pi(k)-1}^{\prime} & \text { if } k+1 \text { is not a prime. }\end{cases}
$$

Suppose (32) is valid for some $k \in I$. Then from (34) and Table 1, we note that $Q(k+1)>(k+1)$ ! whenever $k+1$ is not a prime. Thus $(32)$ is valid for all $k \in I$ if it is valid for all the primes in $I$. There are 301 primes in $I$ 
and (33) is checked to be valid for all these primes. Thus (32) is valid for $k \geq 11380$. On the other hand, we see from $\left|S_{1}\right| \geq t-\pi(k), t \geq t_{0},(3)$ and (11) that

$$
Q(k) \leq \prod_{a_{i} \in S_{1}} a_{i} \leq(k-1) !
$$

This is a contradiction. Thus $k<11380$. Using the lower bounds for $a_{i}$ given by $[3,(15),(16)]$, Erdős and Selfridge obtained $k \leq 30000$. In fact, these lower bounds yield $k \leq 30600$ and an application of the preceding argument sharpens to $k \leq 30000$.

It remains to prove Theorem 1 for $k<11380$. First, let $d=1$. Using (19) we check that

$$
\left\{\begin{array}{l}
f_{0}(k, 2) \geq 4,4 \leq k \leq 22 ; f_{0}(k, 3) \geq 8,23 \leq k \leq 102 \\
f_{0}(k, 4) \geq 16,103 \leq k \leq 282 ; f_{0}(k, 5) \geq 22,283 \leq k \leq 612 \\
f_{0}(k, 6) \geq 38,613 \leq k \leq 1102 ; f_{0}(k, 7) \geq 66,1103 \leq k \leq 1636 \\
f_{0}(k, 8) \geq 115,1637 \leq k \leq 2238
\end{array}\right.
$$

Hence

$$
\left(\begin{array}{c}
f_{0}(k, m)+l-2 \\
l-1
\end{array}\right)>l^{m}
$$

for $l=3, k, m$ chosen as in (35). We note by induction on $l$ that (36) is valid for all $l>3, k, m$ as in (35) since

$$
f_{0}(k, m)>m+1+\frac{3 m(m-1)}{2(9-m)}
$$

and hence

$$
f_{0}(k, m)+l-1>l\left(1+\frac{m}{l}+\left(\begin{array}{c}
m \\
2
\end{array}\right) \frac{1}{l^{2}}+\ldots\right)=l\left(1+\frac{1}{l}\right)^{m},
$$

thereby showing that

$$
\left(\begin{array}{c}
f_{0}(k, m)+l-1 \\
l
\end{array}\right)>(l+1)^{m}
$$

by (36). But this contradicts (24) by (19). Thus we may assume that $k \geq 2239$.

In Table 2, we give the values of $m_{1}, m_{2}$, the range of $k$ and using the definition of $F_{0}\left(k, m_{1}, m_{2}\right)$ from $(22)$ a lower bound for $F_{0}\left(k, m_{1}, m_{2}\right)$, say $F_{0}^{*}\left(m_{1}, m_{2}\right)$, for that range of $k$. 
Table 2

\begin{tabular}{cccc}
\hline$m_{1}$ & $m_{2}$ & $k$ & $F_{0}^{*}\left(m_{1}, m_{2}\right)$ \\
\hline 4 & 11 & $2239-2808$ & 112 \\
4 & 12 & $2809-2960$ & 121 \\
5 & 11 & $2961-3480$ & 195 \\
5 & 12 & $3481-3720$ & 210 \\
5 & 13 & $3721-4488$ & 226 \\
5 & 14 & $4489-5040$ & 241 \\
5 & 15 & $5041-5165$ & 257 \\
6 & 14 & $5166-5328$ & 418 \\
6 & 15 & $5329-6240$ & 445 \\
6 & 16 & $6241-6888$ & 472 \\
6 & 17 & $6889-7920$ & 499 \\
6 & 18 & $7921-9408$ & 526 \\
6 & 19 & $9409-10200$ & 553 \\
6 & 20 & $10201-10608$ & 580 \\
6 & 21 & $10609-11379$ & 607 \\
\hline
\end{tabular}

We check that

$$
\left(\begin{array}{c}
F_{0}^{*}\left(m_{1}, m_{2}\right)+l-2 \\
l-1
\end{array}\right)>l^{m_{1}}\left(\begin{array}{c}
l+m_{2}-1 \\
l-1
\end{array}\right)
$$

for $l=3, m_{1}, m_{2}, F_{0}^{*}\left(m_{1}, m_{2}\right)$ as in Table 2. Since

$$
F_{0}^{*}\left(m_{1}, m_{2}\right)>1+m_{1}+m_{2}+\frac{m_{1} m_{2}}{3}+\frac{m_{1}\left(m_{1}-1\right)\left(m_{2}+3\right)}{2\left(9-m_{1}\right)},
$$

we have

$$
F_{0}^{*}\left(m_{1}, m_{2}\right)+l-1>\left(l+m_{2}\right)\left(1+\frac{1}{l}\right)^{m_{1}}
$$

and hence the inequality

$$
\left(\begin{array}{c}
F_{0}^{*}\left(m_{1}, m_{2}\right)+l-2 \\
l-1
\end{array}\right)>l^{m_{1}}\left(\begin{array}{c}
l+m_{2}-1 \\
l-1
\end{array}\right)
$$

is valid for all $l>3, m_{1}, m_{2}, F_{0}^{*}\left(m_{1}, m_{2}\right)$ as in Table 2 . This contradicts (25) in view of (22). Thus Theorem 1 is valid for $d=1$.

Let $d>1$ and $k<11380$. We first prove Theorem 1(a). Let $d$ be as in (4). By Lemma 2(a), $t^{\prime}=t$ and hence $a_{i}^{\prime}$ are distinct for $1 \leq i \leq t$. Let $l \geq 5$. Then we observe that the hypothesis of Lemma 4 is valid with

$$
l^{\prime}= \begin{cases}l-3 & \text { for } k \geq 11, d \text { even and } k \geq 6, d \text { odd, } \\ l-2 & \text { for } k \geq 121, d \text { even and } k \geq 37, d \text { odd } .\end{cases}
$$

We use (20), (21) and (35) to obtain

$$
\left(\begin{array}{c}
g(k, m-1)+l^{\prime}-1 \\
l^{\prime}
\end{array}\right)>l^{m-1}
$$


with $m$ chosen as in (35) for $k \leq 2238$. This contradicts (26) with $m^{\prime}=m-1$.

Let $k>2238$. We use $G(k) \geq G_{0}\left(k, m_{1}^{\prime}, m_{2}^{\prime}\right),(23)$ and Table 2 to obtain

$$
\left(\begin{array}{c}
G(k)+l^{\prime}-1 \\
l^{\prime}
\end{array}\right)>l^{m_{1}-1}\left(\begin{array}{c}
l^{\prime}+m_{2} \\
l^{\prime}
\end{array}\right)
$$

with $m_{1}$ and $m_{2}$ chosen as in Table 2 . This contradicts (27) since $m_{1}^{\prime} \leq$ $m_{1}-1$ and $m_{2}^{\prime} \leq m_{2}$. Thus we may assume that $3 \leq k \leq 10, d$ even or $3 \leq k \leq 5, d$ odd, $3 \mid d$. Suppose that $3 \leq k \leq 10, d$ even. The number of $a_{i}^{\prime}$ divisible by $p_{1}^{\prime}(\geq 3)$ is at most 4 if $k=10 ; 3$ if $k=7,8,9 ; 2$ if $k=4,5,6$ and 1 if $k=3$. From (20) we find that the number of $a_{i}^{\prime}$ divisible by $p_{1}^{\prime}$ is at least 6 if $k=10 ; 4$ if $4 \leq k \leq 9$; and 3 if $k=3$. This is a contradiction. Suppose that $3 \leq k \leq 5, d$ odd, $3 \mid d$. The number of $a_{i}^{\prime}$ divisible by $p_{1}^{\prime}(\geq 2)$ is at most 3 if $k=5 ; 2$ if $k \in\{3,4\}$; while by (20), this number is at least 4 if $k=5 ; 3$ if $k \in\{3,4\}$ since $3 \mid d$. This contradiction proves Theorem 1(a) for $l \geq 5$.

Let $l=3$. We take $l^{\prime}=2$ and $d \in\{2,3,4,6\}$. The hypothesis of Lemma 4 is valid for $k \geq 40$ if $d \in\{2,3,4\}$ and for $k \geq 100$ if $d=6$. For $k \leq 2238$, we use (20), (21) and (35) to obtain

$$
\left(\begin{array}{c}
g(k, m-1)+1 \\
2
\end{array}\right)>3^{m-1}
$$

with $m$ chosen as in (35) and this contradicts (26) with $m^{\prime}=m-1$. For $k>2238$, we use (23) and Table 2 to obtain

$$
\left(\begin{array}{c}
G(k)+1 \\
2
\end{array}\right)>l^{m_{1}-1}\left(\begin{array}{c}
m_{2}+2 \\
2
\end{array}\right)
$$

with $m_{1}$ and $m_{2}$ chosen as in Table 2. This contradicts (27) since $m_{1}^{\prime} \leq$ $m_{1}-1, m_{2}^{\prime} \leq m_{2}$.

Thus we may suppose that $k \leq 39$ if $d \in\{2,3,4\}$ and $k<100$ if $d=6$. We know that $a_{i}^{\prime}$ for $1 \leq i \leq t$ are cube free. Hence $g(k, 1) \leq 3$ and $g(k, 2)$ $\leq 9$. We check using (21) and (19) that $g_{0}(k, 1) \geq 4$ for $4 \leq k \leq 40$, $g_{0}(k, 2) \geq 10$ for $41 \leq k<100$ if $d=6$ since in this case $g_{0}(k, 2) \geq f_{0}(k, 4)$. Thus we may assume that $k=3$. If $d=2$, 4 , then either $a_{i}^{\prime}=1$ or $3 \mid a_{i}^{\prime}$ for $1 \leq i \leq 3$. This is a contradiction since at most one $a_{i}^{\prime}$ is divisible by 3 and $a_{i}^{\prime}$ are distinct. If $d=3$, then either $a_{i}^{\prime}=1$ or $2 \mid a_{i}^{\prime}$ for $1 \leq i \leq 3$. Hence $n=2 y_{1}^{3}, n+3=y_{2}^{3}, n+6=4 y_{3}^{3}$ or $n=4 y_{1}^{3}, n+3=y_{2}^{3}, n+6=2 y_{3}^{3}$ for some positive integers $y_{1}, y_{2}, y_{3}$. This means $y_{2}^{6}=(n+3)^{2}=\left(2 y_{1} y_{3}\right)^{3}+9$, which is not possible since two cubes $>1$ cannot differ by 9 . If $d=6$, then $a_{1}^{\prime}=a_{2}^{\prime}=a_{3}^{\prime}=1$, which is a contradiction. This completes the proof of Theorem 1(a) for $l=3$.

Now we prove Theorem 1 (b) for $k<11380$. Let $d=5$ and $k \geq 4$. First, we consider the case $l \geq 5$. We observe that the hypothesis of Lemma 4 is 
valid with $l^{\prime}=l-2$ for $k \geq 5$. We note from (20) and (19) that

$$
\begin{cases}g(k, m-1) \geq g_{0}(k, m-1) \geq f_{0}(k, m) & \text { for } m \geq 3 \\ g(k, 2) \geq 5 & \text { for } 5 \leq k \leq 22 .\end{cases}
$$

We use (37) and (35) to obtain

$$
\left(\begin{array}{c}
g(k, 2)+l^{\prime}-1 \\
l^{\prime}
\end{array}\right) \geq l^{2} \quad \text { for } 5 \leq k \leq 22
$$

and

$$
\left(\begin{array}{c}
g(k, m-1)+l^{\prime}-1 \\
l^{\prime}
\end{array}\right)>l^{m-1} \quad \text { for } 23 \leq k \leq 2238
$$

with $m$ chosen as in (35). This contradicts (26).

Let $k>2238$. We use (23) and Table 2 to obtain

$$
\left(\begin{array}{c}
G(k)+l^{\prime}-1 \\
l^{\prime}
\end{array}\right)>l^{m_{1}-1}\left(\begin{array}{c}
l^{\prime}+m_{2} \\
l^{\prime}
\end{array}\right),
$$

which contradicts (27) since $m_{1}^{\prime} \leq m_{1}-1$ and $m_{2}^{\prime} \leq m_{2}$ for $m_{1} \geq 3$ as $5 \nmid a_{i}^{\prime}$ for $1 \leq i \leq t$. Thus we may assume that $k=4$. It is not possible to apply Lemma 4 with $l^{\prime}=l-2$ since the assumption $d \leq k^{l-l^{\prime}-1}$ with $l^{\prime}=l-2$ of Lemma 4 is not valid for $d=5$ and $k=4$. But we observe that $g(k, 2)=4$ and by (7), $n \geq 7^{l}-15>6^{l}$. By following the proof of Lemma 4 , we find that Lemma 4 holds with $l^{\prime}=l-2$. We check that

$$
\left(\begin{array}{c}
g(k, 2)+l-3 \\
l-2
\end{array}\right)>l^{2} \quad \text { for } l \geq 7 .
$$

This contradicts (26). Thus $l=5$. Let $n \geq(12.5)^{5}$. Then we use the upper bound $60 n^{3}+6 \cdot 15^{2} n^{2}+4 \cdot 15^{3} n+15^{4}$ for $\left(n+d_{i_{1}} d\right) \ldots\left(n+d_{i_{l^{\prime}}} d\right)-$ $\left(n+d_{j_{1}} d\right) \ldots\left(n+d_{j_{l^{\prime}}} d\right)$ to see that the assertion of Lemma 4 holds with $l^{\prime}=l-1$. Hence

$$
\left(\begin{array}{c}
g(k, 2)+l-2 \\
l-1
\end{array}\right)>l^{2}
$$

which contradicts (26). Thus we may assume that $n<(12.5)^{5}$. We apply the Algorithm to get $c=298.04<4^{5}, \theta=2, \eta=2, q_{1}=2, q_{2}=3, q_{3}=7, q_{4}=$ 11 and $D=\left\{7^{5}, 2 \cdot 7^{5}, 3 \cdot 7^{5}, 4 \cdot 7^{5}, 6 \cdot 7^{5}, 8 \cdot 7^{5}, 9 \cdot 7^{5}, 12 \cdot 7^{5}, 16 \cdot 7^{5}, 18 \cdot 7^{5}, 11^{5}\right\}$. We take $j=1$ for every $q \in D$ to check Step 3 . Hence by Lemma 11, assumption (7) does not hold, which is a contradiction. This proves Theorem 1(b) for $l \geq 5$.

Let $l=3$ and $l^{\prime}=2$. The hypothesis of Lemma 4 is valid for $k>100$ and we argue as in the case $l=3$ of Theorem 1(a) to exclude the cases $100<k<11380$. Thus $k \leq 100$. Now we use the estimate $n>\gamma(k, 3) k^{3}$ of 
Lemma 3 and Lemma 1(iii) in the proof of Lemma 3 to obtain for $k \geq 79$,

$$
\begin{aligned}
\left|\left\{A_{i} \mid 1 \leq i \leq t, X_{i} \neq 1\right\}\right| & \geq k-1-\pi(k)-\frac{k \log k}{3 \log k+\log \gamma(k, 3)} \\
& \geq(.5681) k .
\end{aligned}
$$

Hence $n>(.5681) k^{4}$. We use this estimate in the inequality $k^{2} d^{2}+2 n k d>$ $3 n^{4 / 3}$ of Lemma 4 to observe that the assertion of Lemma 4 is valid whenever

$$
d \leq \frac{3}{2}(.5681 k)^{1 / 3}-\frac{1}{2 k} .
$$

We use (37) and (35) with $m=3$ to check that

$$
\left(\begin{array}{c}
g(k, 2)+1 \\
2
\end{array}\right)>3^{2} \quad \text { for } 79 \leq k \leq 100
$$

which contradicts (26) with $l^{\prime}=m^{\prime}=2$. Thus $k<79$. Now we check using (20) that $g_{0}(k, 2) \geq 10$ for $25 \leq k<79, k \in\{21,22\}$ and this is not possible since $a_{i}^{\prime}$ are cube free.

Thus we are left with $4 \leq k \leq 20$ and $k \in\{23,24\}$. We see that if $n>40 k^{3}$, then the hypothesis of Lemma 4 is satisfied. Further, $g(k, 2) \geq 4$ and hence

$$
\left(\begin{array}{c}
g(k, 2)+1 \\
2
\end{array}\right)>3^{2}
$$

which contradicts (26) with $l^{\prime}=m^{\prime}=2$. Thus we may assume $n \leq 40 k^{3}$. As earlier, we apply the Algorithm to eliminate the cases $4 \leq k \leq 20$ and $k \in$ $\{23,24\}$. We illustrate the case $k=7$. Then $n+(k-1) d \leq 13750, c=40.1$ $<7^{3}, \theta=3, \eta=5, q_{1}=2, q_{2}=3, q_{3}=7, q_{4}=11, q_{5}=13, q_{6}=17$, $q_{7}=19, q_{8}=23, D=\left\{11^{3}, 2 \cdot 11^{3}, 3 \cdot 11^{3}, 4 \cdot 11^{3}, 6 \cdot 11^{3}, 7 \cdot 11^{3}, 8 \cdot 11^{3}, 9 \cdot 11^{3}\right.$, $\left.13^{3}, 2 \cdot 13^{3}, 3 \cdot 13^{3}, 4 \cdot 13^{3}, 6 \cdot 13^{3}, 17^{3}, 2 \cdot 17^{3}, 19^{3}, 2 \cdot 19^{3}, 23^{3}\right\}$. We check that Step 3 is valid with $j=1$ whenever $q \in D$ but $q \notin\left\{6 \cdot 11^{3}, 3 \cdot 13^{3}, 19^{3}\right\}$ and with $j=2$ otherwise. Hence by Lemma 11, assumption (7) does not hold, which is a contradiction. This completes the proof of Theorem 1(b).

Proof of Corollary 1 . We observe from the equation of Corollary 1 that

$$
\operatorname{ord}_{p}(n(n+d) \ldots(n+(k-1) d)) \equiv 0(\bmod l)
$$

for every prime $p$. We apply the result of Shorey and Tijdeman [9] to deduce that $n(n+d) \ldots(n+(k-1) d)$ is divisible by a prime exceeding $k$ for $1<$ $d \leq 6$. When $d=1$ and $n \leq k$, by Bertrand's postulate, there exists a prime $p$ with $n \leq(n+k) / 2 \leq p<n+k$. Then $p$ divides $n(n+1) \ldots(n+k-1)$ only to the first power, which contradicts (38). Thus we may suppose that $n>k$ whenever $d=1$. Then by a theorem of Sylvester, there exists a prime exceeding $k$ dividing $n(n+1) \ldots(n+k-1)$. Now we apply Theorem 1 to get a contradiction to (38) except in the cases $k=3, d \in\{1,5\}$. To deal 
with these cases, we write as usual $n=a_{1} x_{1}^{l}, n+d=a_{2} x_{2}^{l}, n+2 d=a_{3} x_{3}^{l}$ where $a_{1}, a_{2}, a_{3}$ are $l$ th power free integers. It follows from the equation of Corollary 1 that $P\left(a_{i}\right) \leq 2$. It is easy to check that $a_{i}^{\prime}$ are distinct. Hence $\left(a_{1}, a_{2}, a_{3}\right) \in\left\{\left(2,1,2^{l-1}\right),\left(2^{l-1}, 1,2\right)\right\}$ and $l \geq 3$. Then $\left(2 x_{1} x_{3}\right)^{l}=$ $n(n+2 d)=(n+d)^{2}-d^{2}=x_{2}^{2 l}-d^{2}$, implying $x_{2}^{2}>2 x_{1} x_{3}$. Hence $\left(2 x_{1} x_{3}+1\right)^{l}-$ $\left(2 x_{1} x_{3}\right)^{l} \leq d^{2}$, showing that $d=5, l=3, x_{1} x_{3}=1$, which is impossible.

Proof of Corollary 2. By our assumption when $d=1$ and by the result of Shorey and Tijdeman [9], we see that $P(n(n+d) \ldots(n+(k-1) d))>$ $k$ for $1 \leq d \leq 6$. Hence by Theorem 1 , there exists a prime $p>k$ such that

$$
\operatorname{ord}_{p}(n(n+d) \ldots(n+(k-1) d)) \not \equiv 0(\bmod l) \text {. }
$$

By the equation in Corollary 2, $p$ divides $n(n+d) \ldots(n+(k-1) d)$ to an order which is $\equiv 0(\bmod l)$ since $P(b) \leq k$. This is a contradiction.

\section{Proofs of Theorems A and B}

Proof of Theorem A. Suppose $n \leq k$. Then there exists a prime $p=p^{(k)}$ with $n \leq(n+k) / 2 \leq k \leq p<n+k$. Therefore $p$ divides $n(n+1) \ldots(n+k-1)$ only to the first power. Hence the theorem follows. We may therefore, assume that $n>k$. Then, by a theorem of Sylvester, there exists a prime $p>k$ dividing $n(n+1) \ldots(n+k-1)$. Now, the theorem follows from Theorem 1 (a) with $d=1, t=k$ whenever $k \geq 4$. Thus we need to consider $k=3$. We assume that $\operatorname{ord}_{p}(n(n+1)(n+2)) \equiv 0(\bmod l)$ for every prime $p \geq 3$. We write $n+i=b_{i} x_{i}^{l}$ where $b_{i}$ is $l$ th power free, $P\left(b_{i}\right) \leq 2$ for $0 \leq i \leq 2$. We see as in Lemma 2(a) that $b_{1}, b_{2}, b_{3}$ are distinct. Hence $l \geq 3$. Then it follows as in Lemma 4 that the products $b_{i_{1}} \ldots b_{i_{l-1}}$ are all distinct. We note that $n$ is even and thus $b_{1}=2, b_{2}=1, b_{3}=2^{\alpha-1}$ or $b_{1}=2^{\alpha-1}, b_{2}=1, b_{3}=2$ for some integer $\alpha$ with $2 \leq \alpha \leq l$. Then we have $\left(b_{1}\right)^{\alpha-1}\left(b_{2}\right)^{l-\alpha}=b_{3}\left(b_{2}\right)^{l-2}$ or $\left(b_{3}\right)^{\alpha-1}\left(b_{2}\right)^{l-\alpha}=b_{1}\left(b_{2}\right)^{l-2}$, respectively. This contradicts the fact that $b_{i_{1}} \ldots b_{i_{l-1}}$ are distinct.

Pro of of Theorem B. Since $n \geq k+1$, the left hand side of the equation in Theorem $\mathrm{B}$ is divisible by a prime exceeding $k$ by a theorem of Sylvester. Hence the hypothesis of Theorem 1 with $d=1$ is satisfied and the assertion follows.

\section{References}

[1] L. E. Dickson, History of the Theory of Numbers, Vol. II, Chelsea, New York, 1952.

[2] P. Erdős, On a Diophantine equation, J. London Math. Soc. 26 (1951), 176-178.

[3] P. Erdös and J. L. Selfridge, The product of consecutive integers is never a power, Illinois J. Math. 19 (1975), 292-301. 
[4] D. H. Lehmer, List of prime numbers from 1 to 10006721, Carnegie Institution of Washington, Publication No. 165, 1914.

[5] D. S. Mitrinović, J. Sandor and B. Cristici, Handbook of Number Theory, Kluwer, 1996.

[6] J. B. Rosser and L. Schoenfeld, Approximate formulas for some functions of prime numbers, Illinois J. Math. 6 (1962), 64-94.

[7] T. N. Shorey and Yu. V. Nesterenko, Perfect powers in products of integers from a block of consecutive integers (II), Acta Arith. 76 (1996), 191-198.

[8] T. N. Shorey and R. Tijdeman, Some methods of Erdös applied to finite arithmetic progressions, in: The Mathematics of Paul Erdős I, R. L. Graham and J. Nešetřil (eds.), Springer, 1997, 251-267.

[9] - - - On the greatest prime factor of an arithmetical progression, in: A Tribute to Paul Erdős, A. Baker, B. Bollobás and A. Hajnal (eds.), Cambridge University Press, 1990, 385-389.

[10] - - - Perfect powers in products of terms in an arithmetical progression, Compositio Math. 75 (1990), 307-344.

School of Mathematics

Tata Institute of Fundamental Research

Homi Bhabha Road

Bombay 400 005, India

E-mail: saradha@math.tifr.res.in

Received on 25.10.1996

and in revised form on 14.3.1997 